\title{
A method for image local-difference visualization
}

\author{
Étienne Baudrier \\ Laboratoire de Mathématiques et Applications, \\ Université de La Rochelle, Avenue Crépeau, 17042 La Rochelle Cedex 1, France \\ etienne.baudrier@univ-lr.fr \\ Alain Riffaud \\ UFR Lettres, Université du Maine, Le Mans, France \\ Alain.Riffaud@univ-lemans.fr
}

\begin{abstract}
As ancient documents are being digitized, systems for retrieving documents or images can now be found in Digital Libraries. With regard to illustrations, the content-based image retrieval is difficult and the user often needs to check visually the similarity of the retrieved images. This process is not entirely reliable since very similar images (impressions, ornamental letters) can actually be printed from distinct stamps. No other visualization method exists than the map of pixelto-pixel gray level difference (PPDMap). We propose here a method that evaluates locally the differences and allows the user to visualize them. It is based on a local adaptation of the Hausdorff distance. Our method performance is successfully compared to the PPDMap and an expert difference evaluation assesses our method reliability.
\end{abstract}

\section{Keywords}

Image comparison, binary images, Hausdorff distance, local dissimilarity measure, visualization, ancient images.

\section{Introduction}

This article addresses visualization of differences for ancient-document graphical parts. The evolution of our society toward a model based on digital content has a deep impact on document preservation. Large resources have been invested on digitization programs for the cultural heritage, including maps, historical documents and manuscripts. It is now possible to reach the graphical parts included in the ancient documents: Digital Libraries often propose separated graphical part databases (e.g. impressions, ornamental cap...) [1]. Some systems propose to index these databases $[6,2,11]$. Once similar images have been retrieved either automatically or manually, it can be difficult to notice visually the relevant differences. The next Figure ? gives an example of two image that look like similar but having differences. These differences can be due to time degradation or because the images have been printed from distinct wooden stamps illustrating the same scene.

It would be interesting to visualize all the local differences. Images can differ for various reasons:

- The state of degradation of impression in the original document.

- The digitization which can cause variations in the gray level, the resolution, etc.

- If there is a binarization step, the method used can cause differences in the binarized images.

- The registration can cause a slight shift or/and rotation resulting in differences in the visualization

- The differences made by the engraver when he reproduces wood stamp.

Among all these differences the interesting ones for historian people are those due to the engraver. The other are perturbations due to degradation of paper and digitalizing process. So visualization methods must be able to minimize the impact of perturbations and then to render the relevant differences. There is no works in the literature dealing with this topic. The only way is to do a classical pixel to pixel comparison between 
images (we talk thereafter about Pixel-to-Pixel value Difference Map "PPDMap"). But this information is very poor and yields numerous false differences. Moreover it is not robust to small deformations that are not significant for the user. We propose in this paper a new method based on a parameter-free local version of the Hausdorff distance (HD) that offers a better visualization than the PPDMap and that can help the user in identifying similar graphical parts. The article layout is as follows: firstly, the method is presented for binary images and also for gray-level images, secondly ground truth and experimental results are proposed and finally we conclude and give our perspectives.

\section{Binary image comparison method}

\subsection{Dissimilarity measure based on the Hausdorff distance}

Among dissimilarity measures over binary images, the Hausdorff distance (HD) has often been used in the content-based retrieval domain and is known to have successful applications in object matching [4] or in face recognition [10]. For finite sets of points, the HD can be defined as [4]:

definition 1 (Hausdorff distance) Given two nonempty finite sets of points $F=\left(f_{1}, \ldots, f_{n}\right)$ and $G=$ $\left(g_{1}, \ldots, g_{m}\right)$ of $\mathbb{R}^{2}$, and an underlying distance $d$, the $H D$ is given by

$$
\begin{aligned}
D_{H}(F, G) & =\max (h(F, G), h(G, F)) \\
\text { where } h(F, G) & =\max _{f \in F}\left(\min _{g \in G} d(f, g)\right),
\end{aligned}
$$

$h(F, G)$ is the so-called directed Hausdorff distance.

The classical HD has good properties but it measures the most mismatched points between $F$ and $G$, and as a consequence it is sensitive to noise [7].

Several modifications of the HD have been proposed to improve it such as: the partial HD [4], the modified HD (MHD) [3], the censored HD [7], the "doubly" modified Hausdorff distance [10], the least trimmed squared HD [9] and the weighted Hausdorff distance [5]. Those improved HD are detailed in [12].

It is noticeable that except for the MHD, at least one arbitrary parameter has to be determined. The parameter must be chosen to make the measure as discriminating as possible. These measures are global and cannot account for local dissimilarities. Indeed, the principle of HD is to be a "max min" distance so the value of the HD between two images is reached for at least one couple of points. But it does not say if the value is reached in several parts or only for one pair of points, which corresponds to different degrees of dissimilarity. These remarks motivate us to define a local and parameter-free HD in the next section.

\subsection{Definition of the windowed Hausdorff distance}

The main reason for the modification is that the HD is not defined for empty sets and this case is possible in a window. Moreover, the obtained measures that are obtained when the window is sliding or growing must be consistent. One solution is to introduce the distance to the window side as it follows:

definition 2 (Windowed Hausdorff distance) Let $F, G$ be two bounded sets of $\mathbb{R}^{2}$. $H D_{W}(F, G)=$ $\max \left(h_{W}(F, G), h_{W}(G, F)\right)$

where there are three cases

$$
\begin{aligned}
& \text { 1. } h_{W}(F, G)= \\
& \max _{f \in F \cap W}\left[\min \left(\min _{g \in G \cap W} d(f, g), \min _{w \in F r(W)} d(f, w)\right)\right] \\
& \text { if } F \cap W \neq \emptyset \text { and } G \cap W \neq \emptyset, \\
& \text { 2. } h_{W}(F, G)=\max _{f \in F \cap W}\left[\min _{w \in F r(W)} d(f, w)\right] \text { if } \\
& \quad F \cap W \neq \emptyset \text { and } G \cap W=\emptyset \text {, }
\end{aligned}
$$

3. 0 if $F \cap W=\emptyset$,

where $\operatorname{Fr}(W)$ stands for the frontier of the set $W$.

\subsection{Window-size choice}

The definition of the windowed HD enables a local distance to be made but it introduces a parameter which is the window size. It can be chosen by the user, or automatically and globally, or locally according to the local surrounding. The following properties of the windowed HD allows one to fix locally the window size and then to evaluate the local dissimilarity.

property 1 (Identity) Let $F, G$ be two bounded sets of points of $\mathbb{R}^{2}$, and $W$ a convex closed subset of $\mathbb{R}^{2}$. $H D_{W}(F, G)=0 \Longleftrightarrow F \cap W=B \cap W$

The following properties need the window $W$ to be a ball.

property 2 (Boundary) Let $x \in \mathbb{R}^{2}$ and $r>0$, and let define $W=B(x, r)$ then $H D_{W}(F, G) \leq H D(F, G)$.

This property ensures that the new pieces of information that are taken into account when the window is enlarged do not reduce the former dissimilaritymeasure value. 
property 3 (growth) Let $V=B\left(x_{v}, r_{v}\right)$ and $W=$ $B\left(x_{w}, r_{w}\right)$ be two close discs such as $V \subset W$ then $H D_{V}(F, G) \leq H D_{W}(F, G)$.

Prop. 2 and 3 ensure that a growing sequence of centered windows gives an increasing and bounded sequence of measure values. It allows a stop criterion to be given for such a growing sequence of windows and to be sure it will be satisfied. The measure obtained when the criterion is satisfied is called local Hausdorff distance and the set of measures obtained when this measure is computed for all the pixels is called Local Distance Map (LDMap). The following paragraph presents the algorithm associated to the stop criterion that has been selected.

\section{Algorithm}

A practical algorithm for the computation of the local HD map is proposed below (alg. 1). It consists of a sliding window whose radius is locally adapted to be optimal. It shows the way to adapt the window to

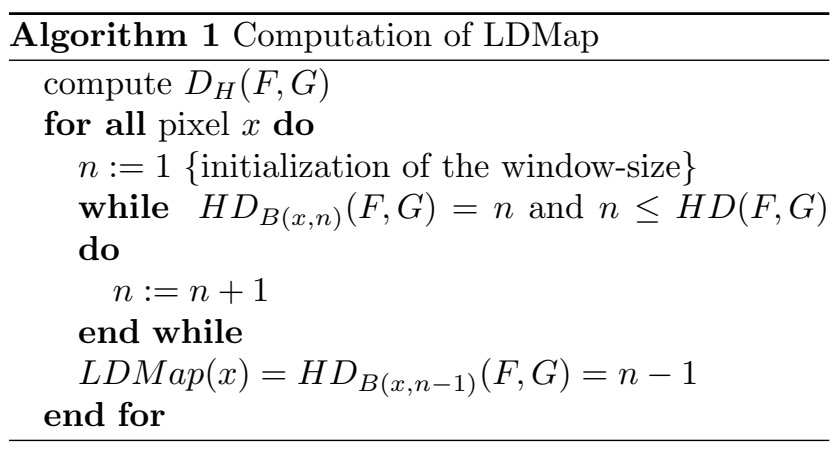

the local dissimilarity: this step is done in the while loop. Nevertheless, this algorithm is time consuming. Indeed, the computation complexity is in $O\left(\mathrm{~m}^{4}\right)$ for two $m \times m$ pixel images. The next section presents a formula for the measure that saves most of the time computation. The computation is faster but the interpretation in terms of local dissimilarity measure comes from algorithm 1.

\subsection{Dissimilarity map}

property 1 (LDMap mathematical formula)

$$
\begin{aligned}
& \forall x \in \mathbb{R}^{2}, \\
& \quad C D L(x)=|B(x)-A(x)| \max (d(x, A), d(x, B))
\end{aligned}
$$

For each pixel $x$, the formula gives a value that depends on the distance transform of the sets $A$ and $B$. Fast algorithms have been developed for distance transformation.So the LDMap complexity with the formula is a $O\left(m^{2}\right)$, which is linear in the pixel number.

\section{The Local Dissimilarity Map for the difference visualization}

In this section we present initial experiments and results about our method. To do it we have constituted in a first step a benchmark of similar images. These images have been manually retrieved from the Ornamental Letters DataBase ${ }^{1}$. Next, as presented in introduction, only the relevant differences should be developed by the visualization method.

To assess the efficiency of ours let's evaluate first its ability to minimize the impact of perturbations and to render the relevant ones. We present these two topics in the next subsections 3.1 and 3.2.

\subsection{Perturbation impact}

This paragraph gives an evaluation of two visualization methods (one based on the LDMap and one using the PPDMap) on monochromatic (gray level) and binary images. As the visualization assessment made by users is not entirely objective, the evaluation of the perturbation rendering is done with a classifier to evaluate the objective part. We have used a database coming from the digital library BVH [8] which includes 168 printings coming from 42 distinct ornamental letter stamps. The database contains four versions of each ornamental letter stamp, coming from four distinct books. The four versions of the same stamp that are available provide some perturbations in the visualization (see Fig 1): perturbations of ageing, digitization, binarization and registration. The tested methods are used to produce maps (see Fig. 2) that are classified by a support vector machine (SVM). The experiment protocol is as follows: the comparison of the 168 images gives 14028 visualization maps that are separated in two classes, one gathering the 252 maps comparing images from the same stamp $C_{\text {sim }}$ and one including the 13876 maps comparing images from dissimilar images $C_{\text {dissim }}$. A SVM learning stage is done on one part of the two classes and a test is realized on the other one. The classification results are compared with those obtained manually. The results, reflecting the average found on 100 tests, are gathered in Tab. 1. Results show that the LDMap allows the SVM to make a better classification than the PPDMap. So the perturbations are less represented in the LDMap than in the PPDMap. The LDMap visualization is therefore better than the PPDMap one. One reason is that a PPDMap does not enable the user to distinguish between a simple translation and a real difference. Let's focus on the LDMap evaluation.

\footnotetext{
${ }^{1}$ http://www.bvh.univ-tours.fr/madonne.asp
} 

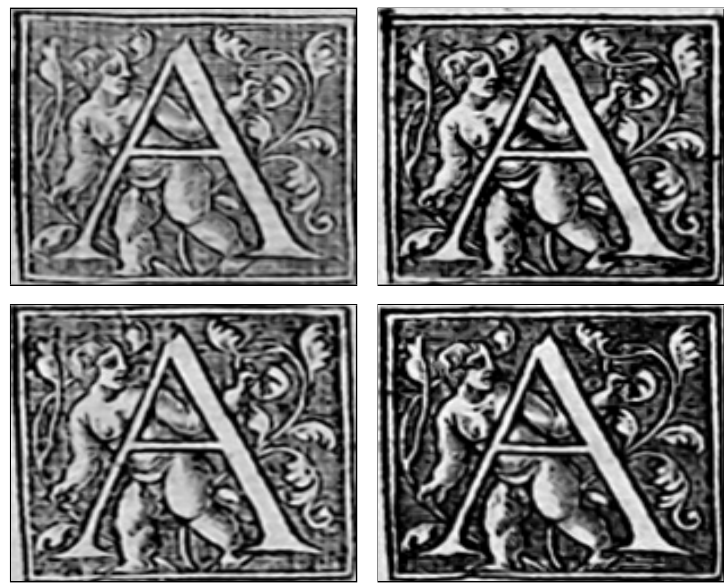

Figure 1. Four impressions from the same stamp with digitization and registration errors.

\begin{tabular}{|l|c|c|}
\hline Successful retrieval & found in $\mathcal{C}_{\text {sim }}$ & found in $\mathcal{C}_{\text {dissim }}$ \\
\hline Gray level LDMap & $\mathbf{9 5 \%}$ & $\mathbf{9 7 \%}$ \\
\hline Binary LDMap & $93 \%$ & $95 \%$ \\
\hline Gray level PPDMap & $70 \%$ & $75 \%$ \\
\hline Binary PPDMap & $70 \%$ & $69 \%$ \\
\hline
\end{tabular}

Table 1. Results of the classification for the gray level and binary LDMap and PPDMap.

By definition, all the differences can be found in the LDMap. The next paragraph is an evaluation of the values used to measure the differences in the LDMap.

\subsection{Expert evaluation of the visualization}

There is no such thing as a perfect visualization: some significant differences are not visually measurable and others, significant according to the user, are very small. Nevertheless it is important to determine if the perturbation measures are inferior than the measures used to find out relevant difference in the LDMap. The experiment is as follows: the test database is composed of 11 pairs of impressions illustrating the same scene but printed from distinct wooden stamps. An expert in ancient graphical documents has circled and evaluated the relevant differences found in the pair of images. The result is the evaluation of 54 relevant differences. These evaluated differences are compared to the values locally obtained in the maps. An example on two ornamental letters "N" is given Fig. 3. The results are presented Fig. 4. The LDMaps have an average devia-

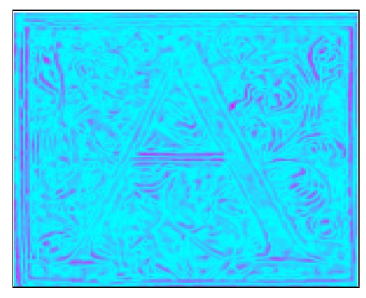

(a) Gray level LDMap

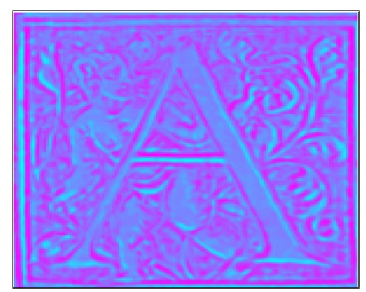

(c) Gray level PPDMap

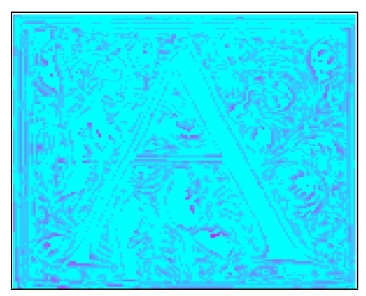

(b) Binary LDMap

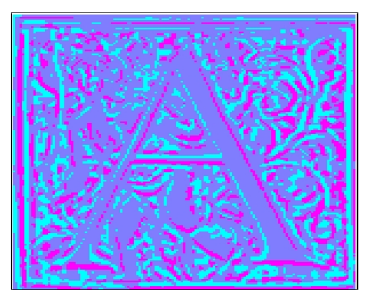

(d) Binary PPDMap
Figure 2. Visualization Maps between the the ornamental letters 1(a) and 1(c). PPDMaps contain more high values than the LDMap: they are more sensitive to pertubations.

tion from the expert evaluation of 1.5 in the binary case and 1.8 in the gray level case. So the binary LDMap has a slightly better behaviour than the gray level one. The graphs show that the LDMap values do not reflect accurately the expert evaluation. But the purpose is to show the user all the relevant differences in the images, even those that are not visually noticeable. Accordingly, the LDMaps present quite reliable values even if they undervalue the relevant differences and do show some perturbations (see Fig. 4).

\section{Conclusion and perspectives}

This article addresses the issue of local difference visualization for the comparison of ancient documents graphical parts. It presents a new image comparison method. It is based on a Hausdorff distance that has been adapted to be windowed. The result is a distance map that gives local dissimilarity measures and their spatial distribution. This map can be fast computed for binary images and a generalization is proposed in the gray level case. A SVM classification based on the LDMap and the PPDMap shows that the LDMap gives less information on the perturbations. Then an expert evaluation of pertinent differences shows that the binary LDMap is more accurate to the expert than the gray level one. It shows also that the LDmap does not reflect the expert evaluation but offers a visualization 


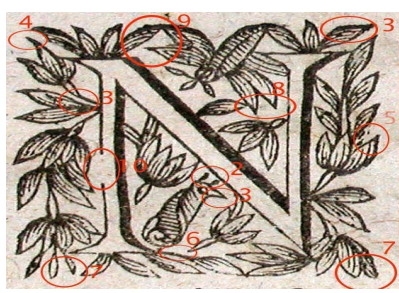

(a) Gray level impression 1 with expert evaluation

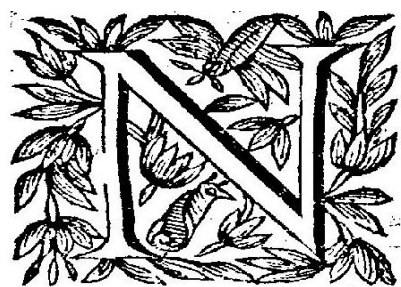

(c) Binarized impression 1

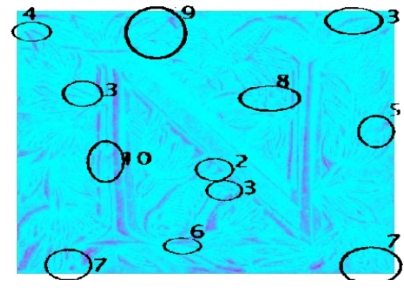

(e) Gray level LDMap

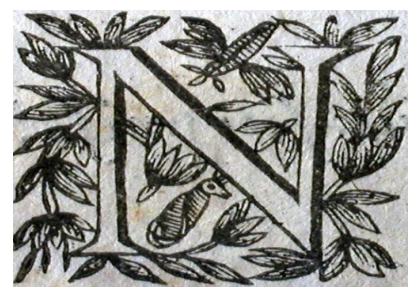

(b) Gray level Impression 2

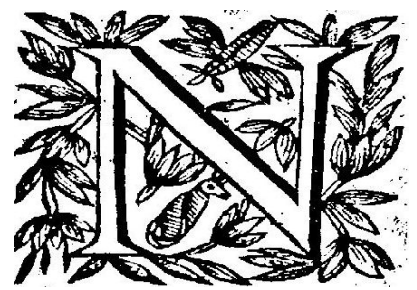

(d) Binarized impression 2

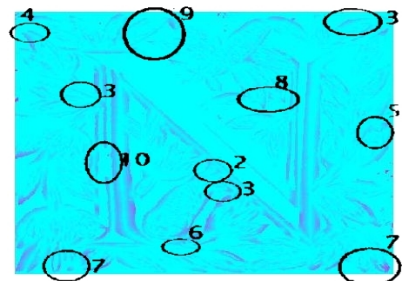

(f) Binary LDMap

Figure 3. A difference evaluation between ornamental letters " $N$ " and a comparison with the visualization maps.

of all the relevant differences.

The next step for us is to test the LDMap over a bigger database, then to implement the LDMap to make it more easily available and to combine LDMap information with higher level information (e.g. connexity) to make it more reliable.

\section{References}

[1] H. S. Baird. Digital library and document image analysis. In Proceedings of the 7th. International Conference on Document Analysis and Recognition ICDAR, pages 1-13. IAPR, 2003.

[2] J. Bigun, S. Bhattacharjee, and S. Michel. Orientation radiograms for image retrieval: an alternative to segmentation. In Proc of international conference on pattern recognition (ICPR), volume 3, pages 346-350. IAPR, 1996.

[3] M.-P. Dubuisson and A. K. Jain. A modified Hausdorff distance for object matching. In Proc. of the International Conference on Pattern Recognition (ICPR), pages 566-568. IAPR, Oct 1994.

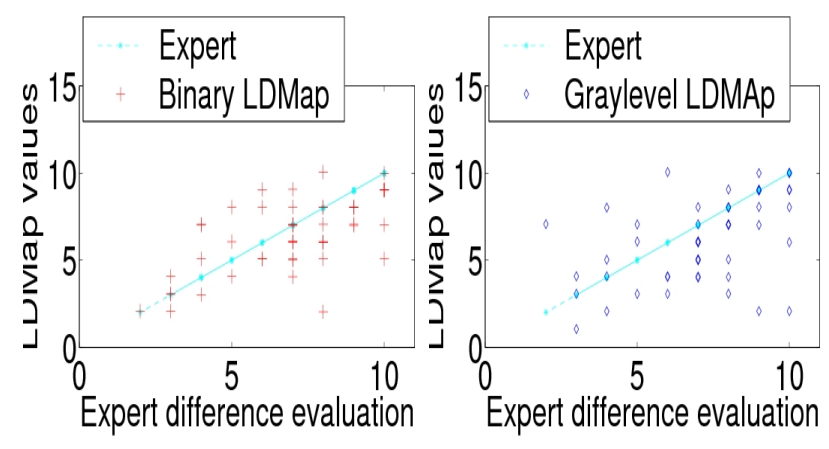

Figure 4. Comparison between the expert pertinent-difference evaluation (in cyan stars) and Left: the binary LDMap (in red); Right: the gray level LDMap (in blue) on 54 differences.

[4] D. P. Huttenlocher, D. Klanderman, and W. J. Rucklidge. Comparing images using the Hausdorff distance. IEEE Transactions on Pattern Analysis and Machine Intelligence, 15(9):850-863, Sep 1993.

[5] Y. Lu, C. Tan, W. Huang, and L. Fan. An approach to word image matching based on weighted Hausdorff distance. In Proc. 6th Internat. Conf. on Document Anal. Recogn., pages 921-925, 10-13 Sep 2001.

[6] R. Pareti, S. Uttama, J. Salmon, J. Ogier, S. Tabbone, L. Wendling, and N. Vincent. On defining signatures for the retrieval and the classification of graphical dropcaps. In Conference on Document Image Analysis for Libraries (DIAL), pages 220-231, 2006.

[7] J. Paumard. Robust comparison of binary images. Pattern Recognition Letters, 18(10):1057-1063, Oct 1997.

[8] J. Ramel, S. Busson, and M. Demonet. Agora: the interactive document image analysis tool of the BVH project. In Conference on Document Image Analysis for Library (DIAL), pages 145-155, 2006.

[9] D.-G. Sim, O.-K. Kwon, and R.-H. Park. Object matching algorithms using robust Hausdorff distance measures. IEEE Transaction on Image Processing, 8(3):425-429, 1999.

[10] B. Takàcs. Comparing faces using the modified Hausdorff distance. Pattern Recognition, 31(12):1873-1881, Dec 1998.

[11] S. Uttama, M. Hammoud, C. Garrido, P. Franco, and J. Ogier. Ancient graphic documents characterization. In Workshop on Graphics Recognition (GREC), pages 97-105, 2006.

[12] C. Zhao, W. Shi, and Y. Deng. A new Hausdorff distance for image matching. Pattern Recognition Letters, 2004 . 